\title{
Multivariate Analysis of the Factors Associated With the Risk of Pneumonia in Intensive Care Units
}

\author{
Cláudia Maria Dantas de Maio Carrilho ${ }^{1}$, Cintia Magalhães Carvalho Grion ${ }^{1}$, Ana Maria Bonametti ${ }^{1}$, \\ Eduardo Alexandrino Servolo Medeiros ${ }^{2}$ and Tiemi Matsuo ${ }^{1}$ \\ ${ }^{1}$ State University of Londrina; Paraná, PR; ${ }^{2}$ Federal University of São Paulo; São Paulo, SP, Brazil
}

\begin{abstract}
Pneumonia is the most frequent hospital infection in patients admitted to intensive care units (ICU) and is also responsible for the highest lethality rates, as well as an increase in both the duration and costs of hospitalization. The objective of this study was to identify predisposing factors for pneumonia. A prospective cohort study was carried out between June 1996 and June 1997, and included 540 patients admitted consecutively for periods greater than 24 hours to the adult ICU of the Londrina State University's Teaching Hospital. Of these, 83 (15.4\%) developed pneumonia. All patients were analyzed with respect to various risk factors for hospital-acquired pneumonia. Univariate analysis identified the following factors: decreased level of consciousness, craniotomy, prior use of antibiotics, mechanical ventilation, nasogastric tube feeding, enteral feeding, aspiration of gastric contents, central venous catheter and the time spent in the ICU. Multivariate analysis identified four risk factors for pneumonia in the ICU: tracheotomy $(R R=1.09 ; 95 \% C I=1.04-1.17)$, nasogastric tube feeding $(R R=1.11 ; 95 \% C I=1.05-1.18)$, H2-blocker use $(R R=1.09 ; 95 \% C I=1.05-1.14)$ and decreased level of consciousness $(R R=2.67 ; 95 \% C I=1.43-5.04)$. In 56.6\% of patients, pneumonia occurred within the first four days following ICU admission. The risk factors identified were all necessary for the treatment of the patient except for decreased level of consciousness, either present at admission or occurring during hospitalization due to deterioration in the clinical condition of the patient or to the use of sedatives.
\end{abstract}

Key-Words: Pneumonia, epidemiology, risk factors.

Hospital infections are one of the most important issues in public health today, and have been aggravated over the past ten years by the emergence of various multiresistant microorganisms such as glycopeptide-resistant staphylococci and enterococci, and carbapenems-resistant Pseudomonas aeruginosa and Acinetobacter baumannii. These infections are among the main causes of in-hospital morbidity and mortality, and result in a significant increase in the mean duration of hospitalization and consequently in higher cost [1].

Hospital-acquired pneumonia occurs at a general rate of $6 \%$; however, in ICUs this rate varies from 10 to $65 \%$ with a mortality rate of $20-70 \%$ [2].

Although all patients hospitalized in ICUs benefit from greater surveillance and more intense medical care, they are also submitted to a greater number of invasive procedures such as venous and urinary catheters, as well as tubes for feeding and mechanical ventilation. These procedures lead to a 5 to 10-fold increase in the incidence of hospital infections and a longer period of hospitalization in the ICU [3].

Various factors predispose the patient to the risk of hospital-acquired pneumonia, and early detection of such factors may alter the focus given to the care of these patients, particularly those at greater risk of developing pneumonia. Among these factors, the individual conditions of the patient should be taken into consideration, such as advanced age, basic diseases, chronic and immunodepressive diseases,

Received on 30 January 2007; revised 15 May 2007.

Address for correspondence: Dr. Claudia Maria Dantas de Maio Carrilho. Rua Antonio Pisicchio 155 apto 104, Londrina, PR, Brazil. Zip Code: 86050-420. Phone: (5543) 33368031. E-mail: carrilho@ sercomtel.com.br.

The Brazilian Journal of Infectious Diseases 2007;11(3):339-344. (C) 2007 by The Brazilian Journal of Infectious Diseases and Contexto Publishing. All rights reserved. abdominal and thoracic surgery, aspiration of gastric contents and therapeutic measures frequently used in patients in the ICU such as antimicrobial medication, endotracheal intubation, vascular catheters, nasogastric tubes, $\mathrm{H} 2$-blockers and enteral feeding. It is important to emphasize that the duration of stay in the ICU and the duration of the procedures are also related to an increase in the occurrence of this infection [4].

Detection of these factors may increase the awareness of the health professionals to the fact that high technology and investment are not always necessary to prevent hospitalacquired pneumonia, since simple routine measures may be implemented for this prevention [5].

The objective of this contemporary cohort study was to evaluate the risk factors for the development of pneumonias in patients admitted to an adult intensive care unit of the State University of Londrina's Teaching Hospital.

\section{Materials and Methods}

This study was carried out at the Teaching Hospital of the State University of Londrina, which has a total of 284 beds including general wards, and neonatal, pediatric and adult ICUs. The adult ICU has two wings (I and II) with a total of 17 beds, where clinical and surgical patients from all sectors of the hospital and from other hospitals in the catchment area receive care. A mean of 98.8 patients are admitted monthly for a mean duration of 6.07 days.

The population evaluated consisted of patients admitted to the adult ICU of the university teaching hospital. The study sample consisted of 540 adult patients admitted consecutively to the ICU between June 1996 and June 1997, who remained in the unit for more than 24 hours. Data were collected concerning identification, diagnosis at admission to the ICU, epidemiology, invasive therapeutic procedures, use of medication and preexistent morbid conditions. All patients were evaluated 
prospectively until reaching one of the study end-points, which were defined as: development of pneumonia, transfer to another department of the hospital, discharge from hospital or death. The patients transferred to another department were followed up for further 48 hours. All patients were submitted to clinical and radiological evaluation at the time of admission to the ICU and thereafter daily during the time they remained in the unit and up to 48 hours following their transfer to another department of the hospital. Clinical data evaluated comprised:

- The presence and duration of use of tracheal tube, respirator, tracheotomy, nebulization with bronchodilator, central venous catheter, urethral catheter, nasogastric tube, enteral feeding and intracranial pressure monitor.

- Aspiration of gastric contents.

- Surgery and the potential for contamination.

- Use and duration of use of corticosteroids, antimicrobial medication, H2-blockers or antacids.

- Presence of concomitant diseases: prior infections, renal failure, liver failure, heart failure, chronic pulmonary disease, neurological dysfunction, diabetes mellitus and obesity.

\section{Definitions}

Hospital-acquired pneumonia was defined according to the clinical/radiological criteria for the diagnosis of hospitalacquired pneumonia established by the Centers for Disease Control and Prevention (CDC) [6].

\section{Statistical Analysis}

The data were collected according to the standardized protocol and analyzed initially using the Epi-Info program, version 6.02 (October 1994), developed by the US Department of Health and Human Services, Public Health Service, Centers for Disease Control and Prevention.

Descriptive statistical analysis included means, standard deviations, confidence intervals for proportions and distributions of the frequencies of variables in the form of tables and graphs.

Initially, a bivariate analysis was carried out on the probable risk factors and the end-point of hospital-acquired pneumonia using the chi-square test. Next, the probable risk factors for hospital-acquired pneumonia were analyzed independently using the stepwise method in the multivariate logistic regression analysis. The risk factors analyzed were the presence of: endotracheal tube, mechanical ventilation, tracheotomy, nasogastric tube, enteral feeding, central venous catheter, surgeries, preexistent clinical conditions (infections, kidney failure, liver failure, heart failure, chronic pulmonary disease, coma, diabetes mellitus and obesity) and the use of corticoids, antimicrobial medication and $\mathrm{H} 2$-blockers.

Significance was established as $5 \%(\mathrm{p}<0.05)$. Results are presented as p-values and relative risks with a $95 \%$ confidence interval.
Descriptive statistical analysis, chi-square test and logistic regression were calculated using the Statistical Analysis System (SAS) software package (SAS Institute, Inc., Cary, North Carolina, USA). The Kaplan-Meier survival curve was calculated to evaluate the time of onset of pneumonia in relation to the risk factor.

\section{Results}

In this cohort of 540 patients, 83 patients developed pneumonia in the ICU at least 48 hours following admission, with an accumulated incidence of $15.4 \%$ and density rate of 20 cases of pneumonia per 1,000 patient-days. Of these patients, $322(59.6 \%)$ were male and 218 (40.4\%) female. A similar distribution was found in the patients who developed pneumonia during the follow-up period. The majority of cases of pneumonia (56.6\%) occurred in the first four days following admission to the ICU.

All the probable risk factors submitted to the bivariate analysis are listed in Table 1 together with the respective p-values, relative risk (RR) and 95\% confidence interval. In this analysis, the following significant risk factors were identified: craniotomy $(\mathrm{RR}=2.27 ; 95 \% \mathrm{CI}=1.34-3.87)$, decreased level of consciousness $(\mathrm{RR}=2.72 ; 95 \% \mathrm{CI}=$ 1.81-4.10), previous use of antibiotics $(\mathrm{RR}=1.73 ; 95 \% \mathrm{CI}$ $=1.16-2.56)$, mechanical ventilation $(\mathrm{RR}=2.80 ; 95 \% \mathrm{CI}=$ 1.59-4.93), nasogastric tube $(\mathrm{RR}=4.00 ; 95 \% \mathrm{CI}=2.17-7.37)$, enteral feeding $(\mathrm{RR}=2.32 ; 95 \% \mathrm{CI}=1.56-3.46)$, aspiration of gastric contents $(\mathrm{RR}=1.93 ; 95 \% \mathrm{CI}=1.07-3.49)$ and central venous catheter $(\mathrm{RR}=2.36 ; 95 \% \mathrm{CI}=1.59-3.53)$. With respect to the time that the patient spent in the ICU, relative risks for a duration of 6-10 days and $>10$ days were, respectively, 4.55 and 11.40 and these risk values were also statistically significant. The duration of hospitalization prior to admission to the ICU, classified as $\leq 10$ days or $>10$ days, was not significant in patients with or without pneumonia.

For the multivariate logistic regression analysis, the stepwise method was used to select the following factors: duration of stay in the ICU, tracheotomy, H2-blockers, nasogastric tube, decreased level of consciousness, heart failure, craniotomy and abdominal surgery. In the analysis of the logistic regression model, the variables whose relative risks were statistically significant, and therefore could be considered to be independent risk factors for the development of pneumonia in patients admitted to the ICU, were: tracheotomy $(\mathrm{RR}=1.09 ; 95 \% \mathrm{CI}=1.04-1.17)$, use of nasogastric tube $(\mathrm{RR}=1.11 ; 95 \% \mathrm{CI}=1.04-1.18)$, use of H2-blockers $(\mathrm{RR}=1.09 ; 95 \% \mathrm{CI}=1.05-1.14)$ and decreased level of consciousness $(\mathrm{RR}=2.67 ; 95 \% \mathrm{CI}=1.43-5.04)$, (Table 2).

Figures 1, 2, 3 and 4 illustrate the accumulated probability of patients with or without tracheotomy, nasogastric tube, decreased level of consciousness and H2-blocker, respectively, not to develop pneumonia in relation to time spent in the ICU (Kaplan-Meier). 
Table 1. Risk factors for hospital-acquired pneumonia in ICU, selected by bivariate analysis

\begin{tabular}{|c|c|c|c|c|c|}
\hline Characteristics & $\begin{array}{c}\text { With } \\
\text { pneumonia } \\
(\mathbf{n}=\mathbf{8 3})\end{array}$ & $\begin{array}{c}\text { Without } \\
\text { pneumonia } \\
(n=457)\end{array}$ & p-value & Relative Risk & $\begin{array}{l}\text { 95\% Confidence } \\
\text { interval }\end{array}$ \\
\hline Gender (male) & $59.0 \%$ & $59.7 \%$ & 0.905 & 1.02 & $0.68-1.53$ \\
\hline Chronic pulmonary disease & $7.2 \%$ & $9.0 \%$ & 0.604 & 1.22 & $0.56-2.65$ \\
\hline Heart failure & $14.5 \%$ & $18.6 \%$ & 0.336 & 1.29 & $0.73-2.29$ \\
\hline Liver failure & $2.4 \%$ & $5.3 \%$ & 0.266 & 2.04 & $0.53-7.80$ \\
\hline Kidney failure & $19.3 \%$ & $22.3 \%$ & 0.537 & 1.17 & $0.70-1.94$ \\
\hline Hemodynamic instability & $41.0 \%$ & $37.0 \%$ & 0.491 & 0.86 & $0.58-1.29$ \\
\hline Imunodepression & $10.8 \%$ & $10.7 \%$ & 0.974 & 0.98 & $0.52-1.86$ \\
\hline Obesity & $1.2 \%$ & $1.5 \%$ & 0.821 & 1.23 & $0.19-7.79$ \\
\hline Diabetes mellitus & $14.5 \%$ & $15.1 \%$ & 0.880 & 1.04 & $0.59-1.83$ \\
\hline Surgery (all) & $28.9 \%$ & $28.4 \%$ & 0.931 & 0.98 & $0.63-1.51$ \\
\hline Laparotomy & $9.6 \%$ & $8.5 \%$ & 0.742 & 1.12 & $0.58-2.18$ \\
\hline Craniotomy & $13.3 \%$ & $5.0 \%$ & 0.004 & 2.27 & $1.34-3.87$ \\
\hline Thoracic & $1.2 \%$ & $5.3 \%$ & 0.153 & 0.25 & $0.04-1.73$ \\
\hline Antimicrobials use & $54.2 \%$ & $69.6 \%$ & 0.006 & 1.73 & $1.16-2.56$ \\
\hline Corticosteroids use & $34.9 \%$ & $26.9 \%$ & 0.135 & 0.72 & $0.48-1.10$ \\
\hline Prior infection & $43.4 \%$ & $53.6 \%$ & 0.086 & 1.41 & $0.95-2.11$ \\
\hline H2-blockers use & $94.0 \%$ & $89.5 \%$ & 0.207 & 1.69 & $0.71-4.00$ \\
\hline Mechanical ventilation & $84.3 \%$ & $62.4 \%$ & 0.001 & & \\
\hline Absent & $15.7 \%$ & $37.6 \%$ & & Reference & - \\
\hline$\leq 3$ days & $44.6 \%$ & $30.4 \%$ & & 2.99 & $1.65-5.44$ \\
\hline$\geq 4$ days & $39.8 \%$ & $31.9 \%$ & & 2.62 & $1.43-4.82$ \\
\hline Nasogastric tube & $86.7 \%$ & $57.5 \%$ & 0.001 & & \\
\hline Absent & $13.3 \%$ & $42.5 \%$ & & Reference & - \\
\hline$\leq 3$ days & $22.9 \%$ & $12.9 \%$ & & 4.54 & $2.26-9.10$ \\
\hline$\geq 4$ days & $63.9 \%$ & $44.6 \%$ & & 3.84 & $2.06-7.17$ \\
\hline Tracheotomy & $13.3 \%$ & $7.9 \%$ & 0.110 & & \\
\hline Absent & $86.7 \%$ & $92.1 \%$ & & Reference & - \\
\hline$\leq 3$ days & $4.8 \%$ & $0.9 \%$ & & 3.42 & $1.66-7.07$ \\
\hline$\geq 4$ days & $8.4 \%$ & $7.0 \%$ & & 1.23 & $0.61-2.49$ \\
\hline Nebulization with bronchodilator & $21.7 \%$ & $20.6 \%$ & 0.817 & 1.05 & $0.65-1.70$ \\
\hline Aspiration of gastric contents & $10.8 \%$ & $5.0 \%$ & 0.039 & 1.93 & $1.07-3.49$ \\
\hline Enteral feeding & $56.6 \%$ & $32.2 \%$ & 0.001 & 2.32 & $1.56-3.46$ \\
\hline Central venous catheter & $57.8 \%$ & $32.8 \%$ & 0.001 & 2.36 & $1.59-3.53$ \\
\hline Decreased level of consciousness & $62.7 \%$ & $34.4 \%$ & 0.001 & 2.72 & $1.81-4.10$ \\
\hline Duration of stay in ICU & & & $<0.001$ & & \\
\hline$\leq 5$ days & $10.8 \%$ & $56.7 \%$ & & Reference & - \\
\hline 6 - 10 days & $24.1 \%$ & $24.3 \%$ & & 4.55 & $2.13-9.71$ \\
\hline$\geq 11$ days & $65.1 \%$ & $19.0 \%$ & & 11.40 & $5.80-22.41$ \\
\hline $\begin{array}{l}\text { Duration of hospitalization } \\
\text { prior to ICU }\end{array}$ & & & 0.177 & & \\
\hline$\leq 10$ days & $81.9 \%$ & $75.1 \%$ & & Reference & - \\
\hline$>10$ days & $18.1 \%$ & $24.9 \%$ & & 1.42 & $0.84-2.40$ \\
\hline
\end{tabular}


Table 2. Risk factors for hospital-acquired pneumonia in ICU, selected by multivariate logistic regression analysis

\begin{tabular}{lccc}
\hline Risk factors & Relative risk & 95\% Confidence interval & Pvalue \\
\hline ICU stay & 0.79 & $0.75-0.84$ & 0.0001 \\
Tracheotomy & 1.09 & $1.04-1.17$ & 0.0008 \\
H2-blockers use & 1.09 & $1.05-1.14$ & 0.0001 \\
Nasogastric tube & 1.11 & $1.04-1.18$ & 0.0004 \\
Decreased level of consciousness & 2.67 & $1.43-5.04$ & 0.0021 \\
Heart failure & 1.98 & $0.87-5.01$ & 0.1173 \\
Craniotomy & 0.33 & $0.12-0.99$ & 0.0343 \\
Abdominal Surgery & 0.41 & $0.16-1.17$ & 0.0590 \\
\hline
\end{tabular}

Figure 1. Kaplan Meyer estimates of pneumonia among the patients with and without tracheotomy.

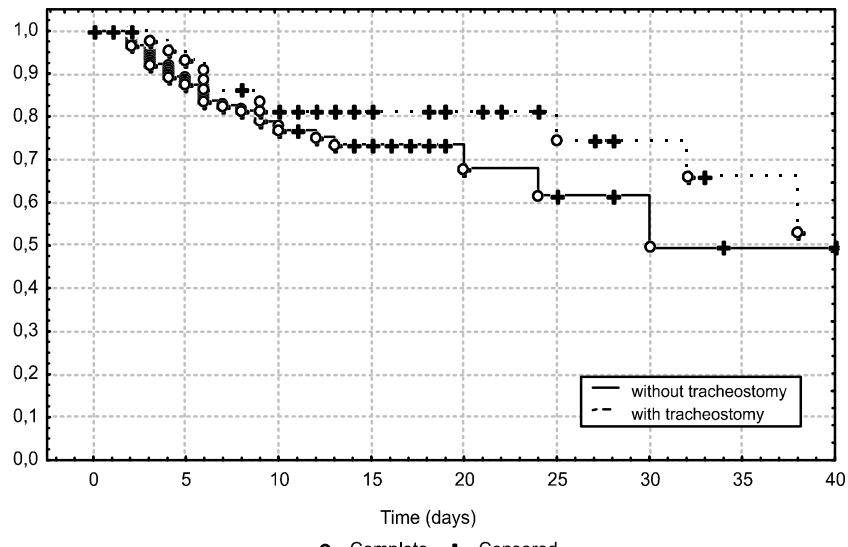

- Complete + Censored

Figure 3. Kaplan Meyer estimates of pneumonia among the patients with and without decreased level of consciousness (coma).

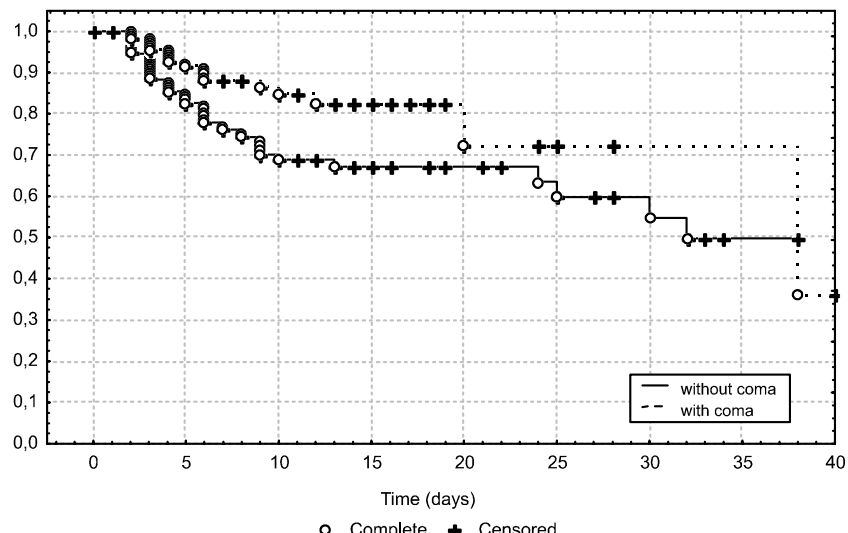

\section{Discussion}

In the period from June 1996 to June 1997, the accumulated incidence and density rates of pneumonia at the university teaching hospital were $15.4 \%$ and 20 cases of pneumonia per 1,000 patient-days, respectively. Pneumonia was the most common infection in this unit according to the data in the report issued by the institution's Commission for the Control
Figure 2. Kaplan Meyer estimates of pneumonia among the patients with and without nasogastric tube (SNG).

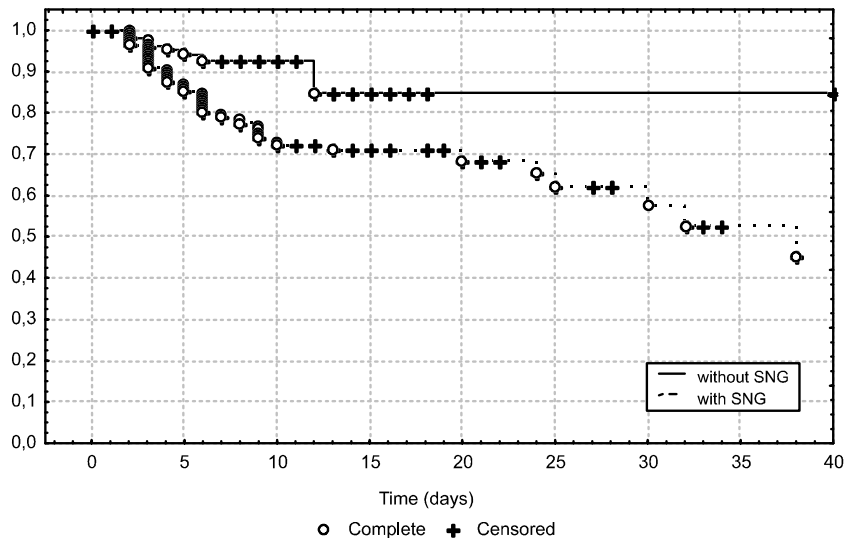

Figure 4. Kaplan Meyer estimates of pneumonia among the patients with and without $\mathrm{H} 2$-blocker.

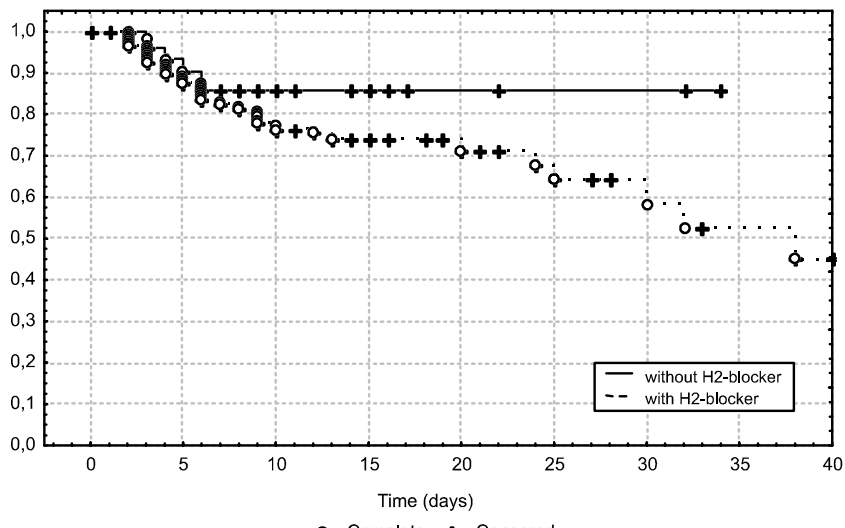

- Complete + Censored

of Hospital Infection. The rate of pneumonia in ICUs may be 10 to 20-fold that found in other departments of the same hospital.

The identification of patients at risk for developing infection is the first step in implementing a surveillance program for hospital-acquired infection. Prior knowledge of potential risk factors for these infections permits implementation of preventive 
measures capable of reducing the incidence and improving the prognosis of these infections.

The risk factors for the development of hospital-acquired pneumonia have been studied using various methodologies that range from a simple descriptive analysis to statistical methods of univariate or multivariate analysis. The principal variables identified as potential risk factors for this hospital infection are: advanced age, changes in cough reflex sensitivity, aspiration of gastric contents, endotracheal intubation, coma, use of intracranial pressure monitor, use of mechanical ventilation and type of ventilation (use of PEEP, for example), use of antacids or H2-blockers, presence of nasogastric tube, tracheotomy, ethylism, enteral feeding, prior use of antimicrobial medication, central nervous system depressants, neurological, thoracic or upper abdominal surgeries, hypoalbuminemia, neuromuscular diseases, chronic obstructive pulmonary disease, bronchoscopy and change of respiratory circuits at intervals of less than 24 hours. The reported incidence of pneumonia in patients on mechanical ventilation may vary between 4 and $45 \%$. Chevret et al. [7] evaluated patients submitted to mechanical ventilation and reported a risk 3.8 times greater of developing pneumonia compared to patients who were not submitted to this procedure. Cunnion et al. [8] analyzed patients admitted to a surgical ICU and observed that these patients had a greater risk of developing pneumonia compared to control patients. These authors also reported that the presence of mechanical ventilation for a period greater than 24 hours increased 12 times the risk of pneumonia compared to patients not artificially ventilated.

Agents that increase gastric $\mathrm{pH}$ also increase the risk of developing pneumonia in patients submitted to mechanical ventilation. The risk of using antacids and $\mathrm{H} 2$-blockers for the development of pneumonia in hospitalized patients has been analyzed in various studies. Donowitz et al. [9] carried out semiquantitative cultures in the gastric fluid of 153 patients in use of cimetidine or antacids. In $47 \%$ of these patients there was a growth of Gram-negative bacilli, while in patients with gastric $\mathrm{pH}<4$, cultures were sterile. A metaanalysis carried out by Messori et al. [10] concluded that ranitidine is ineffective in preventing gastrointestinal bleeding and increases the risk of pneumonia.

Driks et al. [11] evaluated patients submitted to mechanical ventilation and observed a two-fold rate of pneumonia in the group receiving $\mathrm{H} 2$-blockers compared to patients using sucralfate. Nevertheless, Markowicz et al. [12] identified the use and duration of use of sucralfate as a risk factor for pneumonia in patients on mechanical ventilation.

The presence of a nasogastric tube encourages gastroesophageal reflux and the consequent aspiration of gastric contents to the lungs, propitiating the installation of pneumonia. The development of pneumonia is associated not only with the presence of nasogastric tube feeding, but the caliber of this device, the infusion of feeding and the position of the patient in the bed are also related to the genesis of this infection [13].
In patients with a decreased level of consciousness, conditions are created that encourage the aspiration of germs to the lower airways, causing pneumonia. In a study using multivariate analyses, Akca et al. [1] identified a decrease in the level of consciousness $(\mathrm{OR}=3.9)$ and the aspiration of large volumes $(\mathrm{OR}=12.7)$ among other factors responsible for hospital-acquired pneumonia.

The risk of pneumonia associated with tracheotomy is the result of aspiration that may occur in the patient. Even when invisible, "silent" aspiration is frequent and represents a risk for the development of pneumonia [14]. Up to $50 \%$ of aspirations in patients with tracheotomy are "silent" and advanced age increases the risk of this occurrence. When tracheotomy is carried out early, the time of mechanical ventilation may be shortened and the incidence of pneumonia reduced. However, Georges et al. [15] suggest that tracheotomy should be postponed in patients on mechanical ventilation and with bronchial colonization to prevent early pneumonia.

The risk factors detected in this study for the development of pneumonia in patients hospitalized in ICUs (tracheotomy, nasogastric tube feeding, use of $\mathrm{H} 2$-blockers and decreased level of consciousness) are considered exogenous factors, i.e. they are all, except for decreased level of consciousness, a result of the need for therapeutic interventions. Implementation of control measures for these factors may reduce the risk of pneumonia in patients hospitalized in ICUs.

The results of this study suggest that the routine use of $\mathrm{H} 2$-blockers in patients admitted to ICUs should be the subject of review. When there is a real need for prophylaxis of gastrointestinal bleeding, it may be preferable to use sucralfate since this agent does not alter gastric $\mathrm{pH}$.

In addition to these observations, it is important to emphasize that simple measures such as hand washing, ensuring that patients are kept in an appropriate position in bed, less frequent change of respiratory circuits (48 hours to 7 days) and the rational use of antibiotics and central nervous system depressants are low-cost and extremely effective in preventing pneumonia in hospitalized patients.

\section{References}

1. Akca O., Koltka K., Uzel S., et al. Risk factors for early-onset, ventilator-associated pneumonia in critical care patients: selected multiresistent versus nonresistent bacteria. Anesthesiology 2000;93(3):638-45.

2. Alp E., Guven M., Yildiz O., et al. Incidence, risk factors and mortality of nosocomial pneumonia in Intensive Care Units: A prospective study. Ann Clin MicrobAntimicrob [serial on the internet], 2004;3:[about 7 p.]. Avaiable from: http://www.annclinmicrob.com/content/3/1/17.

3. Fagon J.Y., Chastre J., Vuagnat A., et al. Nosocomial pneumonia and mortality among patients in intensive care units. JAMA 1996;275(11):866-9.

4. Bonten M.J., Gaillard C.A., Wounters E.F.M., et al. Problems in diagnosing nosocomial pneumonia in mechanically ventilated patients: a review. Crit Care Med 1994;22(10):1683-91.

5. Kollef M.H. Prevention of hospital-associated pneumonia and ventilator-associated pneumonia. Crit Care Med 2004;32:1396-404. 
6. Garner J.S., Jarvis W.R., Emori T.G., et al. CDC definitions for nosocomial infections. Am J Infect Control 1988;16(3):12840. Erratum in: Am J Infect Control 1988;16(4):177.

7. Chevret S., Hemmer M., Carlet J., Langer M. Incidence and risk factors of pneumonia acquired in intensive care units: results from a multicenter prospective study on 996 patients. Intensive Care Med 1993; 19:256-64.

8. Cunnion K.M., Weber D.J., Broadhead W.E., et al. Risk factors for nosocomial pneumonia: comparing adult critical-care populations. Am J Respir Crit Care Med $1996 ; 153(1): 158-62$.

9. Donowitz L.G., Page M.C., Mileur B.L., Guenthner S.H. Alteration of normal gastric flora in critical care patients receiving antacids and cimetidine therapy. Infect Control 1986;7(1):23-6.

10. Messori A., Trippoli S., Vaiani M., et al. Bleeding and pneumonia in intensive care patients given ranitidine and sucralfate for prevention of stress ulcer: meta-analysis of randomized controlled trials. BMJ 2000;321(7269):1103-06.
11. Driks M.R., Craven D.E., Celli B.R., et al. Nosocomial pneumonia in intubated patients given sucralfate as compared with antacids or histamine type 2 blockers - the role of gastric colonization. New Engl J Med 1987;317(22):1376-82.

12. Markowicz P., Wolff M., Djedaini K., et al. Multicenter prospective study of ventilator-associated pneumonia during acute respiratory distress syndrome. Incidence, prognosis, and risk factors. ARDS Study Group. Am J Respir Crit Care Med 2000;161(6):1942-8.

13. Apostolopoulou E., Bakakos P., Katostaras T., Gregorakos L. Incidence and risk factors for ventilator-associated pneumonia in 4 multidisciplinary intensive care units in Athens, Greece. Respir Care 2003;48(7):681-8.

14. Chastre J., Fagon J.Y. Ventilator-associated pneumonia. Am J Respir Crit Care Med 2002; $165: 867-903$.

15. Georges H., Leroy O., Guery B., et al. Predisposing factors for nosocomial pneumonia in patients receiving mechanical ventilation and requiring tracheotomy. Chest 2000;118(3):767-74. 